Polymer Journal, Vol. 39, No. 5, pp. 423-427 (2007)

(C) 2007 The Society of Polymer Science, Japan

\title{
Temperature Coefficients of Unperturbed Chain Dimensions for Polystyrene and Poly $(\alpha$-methylstyrene)
}

\author{
Masashi Osa, Hidetsugu Kanda, Takenao YoshIZAKI, ${ }^{\dagger}$ and Hiromi YamaKawa \\ Department of Polymer Chemistry, Kyoto University, Katsura, Kyoto 615-8510, Japan
}

(Received November 13, 2006; Accepted February 5, 2007; Published March 15, 2007)

\begin{abstract}
The temperature coefficient $\mathrm{d} \ln \left\langle R^{2}\right\rangle_{0} / \mathrm{d} T$ of the unperturbed mean-square end-to-end distance $\left\langle R^{2}\right\rangle_{0}$ with $T$ the absolute temperature was determined from light scattering measurements for atactic polystyrene (a-PS) with the fraction of racemic diads $f_{\mathrm{r}}=0.59$ and atactic poly $(\alpha$-methylstyrene) (a-P $\alpha \mathrm{MS})$ with $f_{\mathrm{r}}=0.72$, both in toluene (good solvent) and in the range of temperature $15.0-55.0^{\circ} \mathrm{C}$, on the assumption that the binary-cluster integral and hence the gyration-radius expansion factor do not depend on $T$. It is then found that $\mathrm{d} \ln \left\langle R^{2}\right\rangle_{0} / \mathrm{d} T$ is definitely negative for a-PS with a weak helical nature but almost vanishes for a-P $\alpha$ MS with a strong helical nature. The results are consistent with the helical wormlike chain theory prediction. [doi:10.1295/polymj.PJ2006154]

KEY WORDS Unperturbed Chain Dimension / Temperature Coefficient / Helical Wormlike Chain / Polystyrene / Poly $(\alpha$-methylstyrene) /
\end{abstract}

In a new framework of polymer solution science on the basis of the helical wormlike (HW) chain model, ${ }^{1,2}$ we have made a series of experimental studies of dilute solution behavior of typical flexible polymers: atactic polystyrene (a-PS) with the fraction of racemic diads $f_{\mathrm{r}}=0.59,{ }^{3}$ atactic poly(methyl methacrylate) (a-PMMA) with $f_{\mathrm{r}}=0.79,{ }^{4}$ polyisobutylene, ${ }^{5}$ poly(dimethylsiloxane) (PDMS), ${ }^{6}$ isotactic PMMA with $f_{\mathrm{r}}=0.01,{ }^{7}$ and atactic poly $(\alpha$-methylstyrene) $\quad$ (a$\mathrm{P} \alpha \mathrm{MS})$ with $f_{\mathrm{r}}=0.72 .{ }^{8}$ From an analysis of experimental data so far obtained for these polymers, including their oligomers, in their respective $\Theta$ states, we have determined their HW model parameters to obtain valuable information about their chain stiffness and local chain conformations from those values so determined. ${ }^{1,8}$ It has then been shown that a-PMMA, PDMS, and a-P $\alpha$ MS have rather large chain stiffness and strong helical nature ${ }^{1}$ that is, these three polymer chains tend to retain rather large and clearly distinguishable helical portions in dilute solution, while the other three do not. In the HW framework, it is predicted that the temperature coefficient of the unperturbed mean-square end-to-end distance $\left\langle R^{2}\right\rangle_{0}$ in the coil limit, which is defined by $\mathrm{d} \ln \left\langle R^{2}\right\rangle_{0} / \mathrm{d} T$ with $T$ the absolute temperature, may vanish or even become positive for flexible polymers having strong helical nature, while it is definitely negative for most of flexible polymers having weak helical nature. ${ }^{1,9}$ The purpose of the present paper is to confirm experimentally this prediction taking as examples a-PS with a weak helical nature and a-P $\alpha$ MS with a strong one.

This temperature coefficient was first investigated by Flory in his study of unperturbed chain dimensions on the basis of the rotational isomeric state (RIS) model. ${ }^{10}$ Specifically, in some cases, its observed values have been used for assignment of proper values of the RIS model parameters (statistical weights) for a given polymer chain, so that the model can necessarily reproduce those values. On the other hand, the HW model parameters have been determined so that the observed dependence on the molecular weight of, for instance, the unperturbed mean-square radius of gyration $\left\langle S^{2}\right\rangle_{0}$ may be well reproduced by the corresponding HW theory. There is therefore no guarantee that the HW model may reproduce well the observed value of the temperature coefficient. The present analysis may then provide an additional evidence of the validity of the HW model.

As for experimental values of $\mathrm{d} \ln \left\langle R^{2}\right\rangle_{0} / \mathrm{d} T$ for a-PS and a-P $\alpha$ MS, Mays et al. ${ }^{11,12}$ have already reported the values $-1.1 \times 10^{-3}$ and $-0.3_{0} \times 10^{-3} \mathrm{deg}^{-1}$, respectively, which have been determined from the intrinsic viscosity in several poor solvents in the vicinity of the respective $\Theta$ temperatures by the use of the Burchard-Stockmayer-Fixman plot. ${ }^{13,14}$ Unfortunately, however, the values of $f_{\mathrm{r}}$ of their a-P $\alpha$ MS samples, almost all of which are commercial ones from Pressure Chemical Co. or Polymer Laboratories, Ltd., may be considered to be different from ours, ${ }^{8,15}$ and therefore we need to carry out our own experimental investigation by the use of our samples for which the HW model parameters have been determined. Further, we use a maneuver to evaluate $\mathrm{d} \ln \left\langle R^{2}\right\rangle_{0} / \mathrm{d} T$ from observed values of the (perturbed) mean-square radius of gyration $\left\langle S^{2}\right\rangle$ determined in a good solvent toluene at several temperatures on the assumption that the binary-cluster integral $\beta$ and hence the gyrationradius expansion factor $\alpha_{S}$ do not depend on temper-

${ }^{\dagger}$ To whom correspondence should be addressed (E-mail: yoshizaki@molsci.polym.kyoto-u.ac.jp). 
Table I. Values of $M_{\mathrm{w}}$ and $M_{\mathrm{w}} / M_{\mathrm{n}}$ for polystyrene and $\operatorname{poly}(\alpha$-methylstyrene $)$

\begin{tabular}{lcc}
\hline polymer (sample code) & $M_{\mathrm{w}}$ & $M_{\mathrm{w}} / M_{\mathrm{n}}$ \\
\hline a-PS (F380) & $3.9_{4} \times 10^{6}$ & 1.05 \\
a-P $\alpha$ MS (AMS360) & $3.6_{1} \times 10^{6}$ & 1.07 \\
\hline
\end{tabular}

ature. We also carry out similar measurements on a-PS to compare the result with Mays et al.'s one. ${ }^{11}$ Then the above assumption is shown to be valid.

\section{EXPERIMENTAL}

\section{Materials}

The a-PS sample F380 is the same as that used in previous studies of $\alpha_{S},{ }^{16}$ the viscosity-radius expansion factor, ${ }^{17}$ the second virial coefficient $A_{2},{ }^{18}$ and the hydrodynamic-radius expansion factor, ${ }^{19,20}$ i.e., the fraction separated by fractional precipitation from the commercial standard sample from Tosoh Co., Ltd., the value of its $f_{\mathrm{r}}$ being 0.59 . The a-P $\alpha \mathrm{MS}$ sample AMS360 used in this work is a fraction newly separated by fractional precipitation from the original sample previously ${ }^{8}$ prepared by living anionic polymerization, and therefore the value of its $f_{\mathrm{r}}$ is 0.72 . The values of the weight-average molecular weight $M_{\mathrm{w}}$ determined from light-scattering (LS) measurements and the ratio of $M_{\mathrm{w}}$ to the number-average molecular weight $M_{\mathrm{n}}$ determined by analytical gel permeation chromatography are given in Table I. We note that $M_{\mathrm{w}}$ of F380 and AMS360 were determined in toluene at $15.0^{\circ} \mathrm{C}$ in the previous work ${ }^{16}$ and in toluene at $25.0^{\circ} \mathrm{C}$ in this work, respectively.

The solvent toluene used for LS measurements was purified according to a standard procedure prior to use.

\section{Light Scattering}

LS measurements were carried out to determine $M_{\mathrm{w}}$ for the a-P $\alpha$ MS sample AMS360 in toluene at $25.0^{\circ} \mathrm{C}$, and $\left\langle S^{2}\right\rangle$ and $A_{2}$ for the a-PS sample F380 and for AMS360, both in toluene at 15.0, 25.0, 35.0, 45.0, and $55.0^{\circ} \mathrm{C}$. A Fica 50 light-scattering photometer was used for all the measurements with vertically polarized incident light of wavelength $436 \mathrm{~nm}$. For a calibration of the apparatus, the intensity of light scattered from pure benzene was measured at $25.0^{\circ} \mathrm{C}$ at a scattering angle of $90^{\circ}$, where the Rayleigh ratio $R_{\mathrm{Uu}}\left(90^{\circ}\right)$ of pure benzene was taken as $46.5 \times 10^{-6}$ $\mathrm{cm}^{-1} .{ }^{21}$ The depolarization ratio $\rho_{\mathrm{u}}$ of pure benzene at $25.0^{\circ} \mathrm{C}$ was determined to be $0.41 \pm 0.01$ by the method of Rubingh and $\mathrm{Yu}^{22}$ Scattered intensities were measured at six different concentrations and at fifteen scattering angles ranging from 15 to $45^{\circ}$. The data obtained were treated by using the Berry square-root plot. ${ }^{23}$

The most concentrated solution of each sample was prepared gravimetrically and made homogeneous by continuous stirring at room temperature for $2 \mathrm{~d}$. It was optically purified by filtration through a Teflon membrane of pore size $0.45 \mu \mathrm{m}$. The solutions of lower concentrations were obtained by successive dilution. The weight fractions of the test solutions were converted to the polymer mass concentrations $c$ by the use of the densities of the respective solutions calculated with the partial specific volumes $v_{2}$ of the samples and with the density $\rho_{0}$ of the solvent toluene. The values of $v_{2}$ used, which were assumed to be independent of temperature in the range from 15.0 to $55.0^{\circ} \mathrm{C}$, are $0.910 \mathrm{~cm}^{3} / \mathrm{g}$ for a-PS in toluene at $15.0^{\circ} \mathrm{C}^{16}$ and $0.875 \mathrm{~cm}^{3} / \mathrm{g}$ for a-P $\alpha \mathrm{PS}$ in toluene at $25.0^{\circ} \mathrm{C} .{ }^{24}$ The values of $\rho_{0}$ used for toluene at 15.0, $25.0,35.0,45.0$, and $55.0^{\circ} \mathrm{C}$ are $0.8704,0.8622$, $0.8531,0.8438$, and $0.8364 \mathrm{~g} / \mathrm{cm}^{3}$, respectively, which were calculated by interpolation of literature values. ${ }^{25}$

The values of the refractive index increment $\partial n / \partial c$ at wavelength of $436 \mathrm{~nm}$ used are $0.110_{8} \mathrm{~cm}^{3} / \mathrm{g}$ for a-PS in toluene at $15.0^{\circ} \mathrm{C}^{16}$ and $0.131_{0} \mathrm{~cm}^{3} / \mathrm{g}$ for $\mathrm{a}-\mathrm{P} \alpha \mathrm{PS}$ in toluene at $25.0^{\circ} \mathrm{C} .{ }^{24}$ For each polymer sample, the values of $\partial n / \partial c$ at the other temperatures were calculated so that they gave the value of $M_{\mathrm{w}}$ determined at the reference temperature (15.0 or $25.0^{\circ} \mathrm{C}$ ). The values of the refractive index $n_{0}$ used for toluene at $15.0,25.0,35.0,45.0$, and $55.0^{\circ} \mathrm{C}$ are $1.5211,1.5151,1.5091,1.5031$, and 1.4971 , respectively, which were calculated from an interpolation formula. ${ }^{26}$

\section{RESULTS AND DISCUSSION}

The values of $\left\langle S^{2}\right\rangle$ and $A_{2}$ determined from LS measurements for the a-PS sample F380 and the a-P $\alpha$ MS sample AMS360 in toluene at 15.0, 25.0, 35.0, 45.0, and $55.0^{\circ} \mathrm{C}$ are given in Table II. We note

Table II. Values of $\left\langle S^{2}\right\rangle$ and $A_{2}$ for polystyrene and poly $(\alpha$-methylstyrene $)$ in toluene

\begin{tabular}{cccc}
\hline sample & $\begin{array}{c}\text { Temp. } \\
\left({ }^{\circ} \mathrm{C}\right)\end{array}$ & $\begin{array}{c}10^{-5}\left\langle\mathrm{~S}^{2}\right\rangle \\
\left(\AA^{2}\right)\end{array}$ & $\begin{array}{c}10^{4} A_{2} \\
\left(\mathrm{~cm}^{3} \mathrm{~mol} / \mathrm{g}^{2}\right)\end{array}$ \\
\hline F380 & 15.0 & $9.8_{5}$ & $2.0_{2}$ \\
& 25.0 & $9.5_{7}$ & $1.9_{2}$ \\
& 35.0 & $9.4_{0}$ & $1.9_{3}$ \\
& 45.0 & $9.2_{9}$ & $1.8_{8}$ \\
& 55.0 & $9.1_{6}$ & $1.8_{7}$ \\
AMS360 & & & \\
& 15.0 & $7.3_{7}$ & $1.6_{1}$ \\
& 25.0 & $7.3_{4}$ & $1.6_{2}$ \\
& 35.0 & $7.3_{0}$ & $1.5_{2}$ \\
& 45.0 & $7.1_{7}$ & $1.5_{3}$ \\
& 55.0 & $7.3_{8}$ & $1.6_{0}$ \\
\hline
\end{tabular}




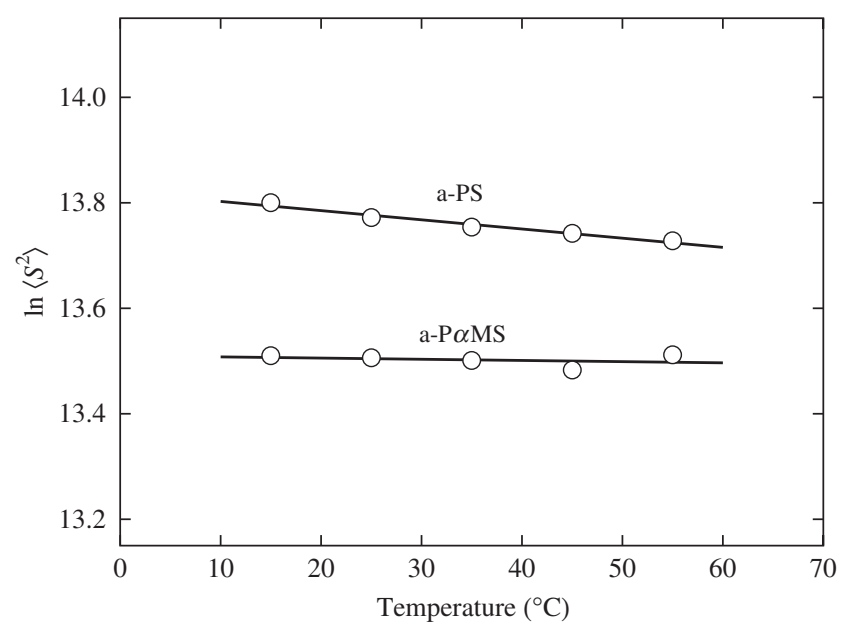

Figure 1. Plots of $\ln \left\langle S^{2}\right\rangle\left(\left\langle S^{2}\right\rangle\right.$ in $\left.\AA^{2}\right)$ against temperature for a-PS and a-P $\alpha \mathrm{MS}$ in toluene.

that the values of $M_{\mathrm{w}}$ for the two samples are so large that the behavior of their (entire) chain dimensions may be well explained by the Gaussian chain. ${ }^{1,16,24}$

Figure 1 shows plots of $\ln \left\langle S^{2}\right\rangle\left(\left\langle S^{2}\right\rangle\right.$ in $\left.\AA^{2}\right)$ against temperature for F380 and AMS360 in toluene. The straight line associated with each polymer is the best-fit one determined by the least-squares method. It is interesting to see that $\ln \left\langle S^{2}\right\rangle$ appreciably decreases with increasing temperature for a-PS but is almost independent of temperature for a-P $\alpha \mathrm{MS}$ in the range of temperature examined.

For perturbed polymer chains with excluded volume, the derivative of $\ln \left\langle S^{2}\right\rangle$ with respect to $T$ may be written in the form,

$$
\frac{\mathrm{d} \ln \left\langle S^{2}\right\rangle}{\mathrm{d} T}=\frac{\mathrm{d} \ln \left\langle S^{2}\right\rangle_{0}}{\mathrm{~d} T}+2 \frac{\mathrm{d} \ln \alpha_{S}}{\mathrm{~d} T}
$$

where we note that $\alpha_{S}$ is a function of $\beta{ }^{1,27}$ It is known that the second virial coefficient $B_{2}$ for gases, which is equivalent to $\beta$, becomes independent of $T$ in the range of high $T$ (above the Boyle temperature), and that such behavior may be well explained by a simple molecular model with the Lennard-Jones 612 potential. ${ }^{28}$ For polymer chains in dilute solution, the statistical segments (or repeat units) constituting the chain may be considered to interact with each other through a potential of mean force roughly of the Lennard-Jones 6-12 type, i.e., a hard repulsive core along with a short-range attractive tail. It may therefore be assumed that $\beta$ and hence $\alpha_{S}$ are independent of $T$ in good solvents, i.e.,

$$
\frac{\mathrm{d} \ln \alpha_{S}}{\mathrm{~d} T} \simeq 0 \quad \text { (in good solvents) }
$$

If $\beta$ is independent of $T$ in good solvents, $A_{2}$ necessarily becomes proportional to $\left\langle S^{2}\right\rangle^{3 / 2}\left(\left\langle S^{2}\right\rangle_{0}{ }^{3 / 2}\right)$ for a given flexible polymer with very large $M_{\mathrm{w}}$ within the
Table III. Values of $\mathrm{d} \ln \left\langle R^{2}\right\rangle_{0} / \mathrm{d} T$

\begin{tabular}{lcc}
\hline & \multicolumn{2}{c}{$10^{3} \mathrm{~d} \ln \left\langle R^{2}\right\rangle_{0} / \mathrm{d} T\left(\mathrm{deg}^{-1}\right)$} \\
\cline { 2 - 3 } polymer $\left(f_{\mathrm{r}}\right)$ & obsd $\left(15.0-55.0^{\circ} \mathrm{C}\right)$ & calcd $(300 \mathrm{~K})$ \\
\hline a-PS $(0.59)$ & $-1.7_{4}$ & -3.21 \\
a-P $\alpha$ MS $(0.72)$ & $-0.2_{2}$ & 0.28 \\
\hline
\end{tabular}

framework of the (quasi-) two-parameter theory. ${ }^{1,27}$ As seen from the third column of Table II, $A_{2}$ slightly decreases with increasing temperature for a-PS but is almost independent of temperature for a-P $\alpha \mathrm{MS}$, being in accordance with the behavior of $\left\langle S^{2}\right\rangle$ mentioned above. This indicates that the above assumption is valid.

Under this assumption, $\mathrm{d} \ln \left\langle R^{2}\right\rangle_{0} / \mathrm{d} T$ may be related directly to $\mathrm{d} \ln \left\langle S^{2}\right\rangle / \mathrm{d} T$ as follows,

$$
\frac{\mathrm{d} \ln \left\langle R^{2}\right\rangle_{0}}{\mathrm{~d} T}=\frac{\mathrm{d} \ln \left\langle S^{2}\right\rangle_{0}}{\mathrm{~d} T} \simeq \frac{\mathrm{d} \ln \left\langle S^{2}\right\rangle}{\mathrm{d} T}
$$

From the slopes of the straight lines shown in Figure $1, \mathrm{~d} \ln \left\langle R^{2}\right\rangle_{0} / \mathrm{d} T$ may then be evaluated for a-PS and a-P $\alpha \mathrm{MS}$ in the range of temperature 15.0$55.0^{\circ} \mathrm{C}$, and the results are given in the second column of Table III.

The value $-1.7_{4} \times 10^{-3} \mathrm{deg}^{-1}$ of $\mathrm{d} \ln \left\langle R^{2}\right\rangle_{0} / \mathrm{d} T$ for a-PS is of the same order of magnitude as the literature value $-1.1 \times 10^{-3} \mathrm{deg}^{-1}$ obtained by Mays et $a l .{ }^{11}$ indicating the validity of the above-mentioned assumption on $\beta$ and $\alpha_{S}$. The value $-0.2_{2} \times 10^{-3}$ $\mathrm{deg}^{-1}$ for our a-P $\alpha$ MS sample $\left(f_{\mathrm{r}}=0.72\right)$ is in good agreement with the literature value $-0.3_{0} \times 10^{-3}$ $\mathrm{deg}^{-1}$ obtained by Mays et al. ${ }^{12}$ for the commercial samples, and they are one order of magnitude smaller than those for a-PS. As seen from Figure 1 and also from Figure 3 of ref 12, it may be said that $\mathrm{d} \ln \left\langle R^{2}\right\rangle_{0} / \mathrm{d} T$ for a-P $\alpha \mathrm{MS}$ vanishes within experimental error.

Now we evaluate $\mathrm{d} \ln \left\langle R^{2}\right\rangle_{0} / \mathrm{d} T$ theoretically on the basis of the HW chain model. The (unperturbed) HW chain ${ }^{1}$ is a general elastic wire model with both bending and torsional energies such that its total elastic (potential) energy becomes a minimum of zero when its contour becomes a regular helix called the characteristic helix, whose shape as a space curve is specified by the differential-geometrical curvature $\kappa_{0}$ and torsion $\tau_{0}$. We note that the bending and torsional force constants may be put equal to each other for flexible polymer chains. ${ }^{1}$ Instead of the common force constant $\alpha$ so introduced, we use the static stiffness parameter $\lambda^{-1}=2 \alpha / k_{\mathrm{B}} T$ with $k_{\mathrm{B}}$ the Boltzmann constant as a measure of chain stiffness. Note that $\lambda^{-1}$ becomes small, or the HW chain becomes flexible, with increasing $T$ if $\alpha$ is independent of $T$. The conformational behavior of the HW chain may then be specified by the three parameters $\kappa_{0}, \tau_{0}$, and $\lambda^{-1}$. (When the de- 
Table IV. Values of the HW model parameters

\begin{tabular}{lccccc}
\hline polymer $\left(f_{\mathrm{r}}\right)$ & solvent & $\begin{array}{c}\text { Temp. } \\
\left({ }^{\circ} \mathrm{C}\right)\end{array}$ & $\lambda^{-1} \kappa_{0}$ & $\lambda^{-1} \tau_{0}$ & $\begin{array}{c}\lambda^{-1} \\
(\AA)\end{array}$ \\
\hline a-PS $(0.59)$ & cyclohexane & 34.5 & 3.0 & 6.0 & 20.6 \\
a-P $\alpha$ MS $(0.72)$ & cyclohexane & 30.5 & 3.0 & 0.9 & 46.8 \\
\hline
\end{tabular}

pendence of dilute solution properties on $M_{\mathrm{w}}$ is discussed, the total contour length $L$ of the HW chain must be converted to $M_{\mathrm{w}}$ by further introducing the shift factor $M_{\mathrm{L}}$ as defined as the molecular weight per unit contour length.)

For the elastic wire model, the temperature coefficients of $L, \kappa_{0}^{-1}$, and $\tau_{0}^{-1}$ must be of the same order of magnitude as linear thermal expansion coefficients of ordinary solids $\left(10^{-6}-10^{-5} \mathrm{deg}^{-1}\right)$, so that their dependence on $T$ may be ignored. Further, we assume that the force constant $\alpha$ is independent of $T$. Then the only quantity that depends on $T$ is $\lambda$, which is proportional to $T$, and $\mathrm{d} \ln \left\langle R^{2}\right\rangle_{0} / \mathrm{d} T$ may be written in the form, ${ }^{1,9}$

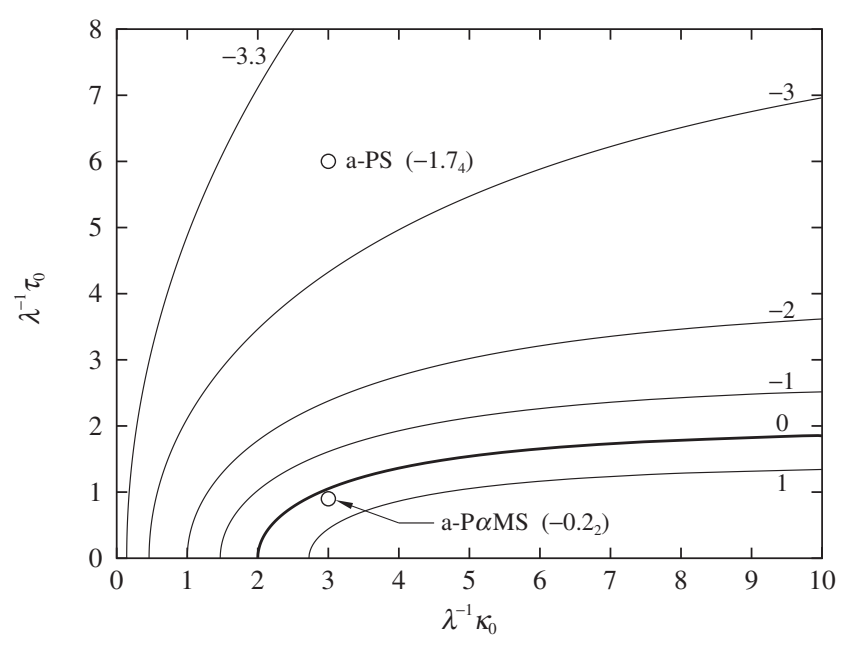

Figure 2. Contour map of $10^{3} \mathrm{~d} \ln \left\langle R^{2}\right\rangle_{0} / \mathrm{d} T$ in a $\left(\lambda^{-1} \kappa_{0}\right.$, $\lambda^{-1} \tau_{0}$ )-plane at $T=300 \mathrm{~K}$. The heavy and light solid curves represent the contour lines for the indicated theoretical values of $10^{3} \mathrm{~d} \ln \left\langle R^{2}\right\rangle_{0} / \mathrm{d} T$, the values in parentheses being the observed ones for the two polymers.

$$
\frac{\mathrm{d} \ln \left\langle R^{2}\right\rangle_{0}}{\mathrm{~d} T}=-\frac{16-4\left(\lambda^{-1} \kappa_{0}\right)^{2}+\left[8+\left(\lambda^{-1} \kappa_{0}\right)^{2}+\left(\lambda^{-1} \tau_{0}\right)^{2}\right]\left(\lambda^{-1} \tau_{0}\right)^{2}}{\left[4+\left(\lambda^{-1} \tau_{0}\right)^{2}\right]\left[4+\left(\lambda^{-1} \kappa_{0}\right)^{2}+\left(\lambda^{-1} \tau_{0}\right)^{2}\right]} T^{-1}
$$

We note that $\mathrm{d} \ln \left\langle R^{2}\right\rangle_{0} / \mathrm{d} T$ is always equal to $-T^{-1}$ (negative) for the Kratky-Porod wormlike chain ${ }^{1,29}$ which is a special case of the HW chain with $\kappa_{0}=0$.

In Table IV are given the values of $\lambda^{-1} \kappa_{0}, \lambda^{-1} \tau_{0}$, and $\lambda^{-1}$ determined from an analysis of $\left\langle S^{2}\right\rangle_{0}$ for a-PS in cyclohexane at $34.5^{\circ} \mathrm{C}(\Theta)^{1,16,30}$ and a-P $\alpha$ MS in cyclohexane at $30.5^{\circ} \mathrm{C}(\Theta) .{ }^{8}$ The values of $10^{3} \mathrm{~d} \ln \left\langle R^{2}\right\rangle_{0} / \mathrm{d} T$ at $300 \mathrm{~K}$ calculated from eq 4 with these HW model parameter values are given in the third column of Table III. Although agreement between theory and experiment is only semiquantitative, the theory explains correctly the difference between a-PS and a-P $\alpha \mathrm{MS}$; that is, $\mathrm{d} \ln \left\langle R^{2}\right\rangle_{0} / \mathrm{d} T$ is definitely negative for the former but almost vanishes for the latter.

A contour map of the temperature coefficient at $300 \mathrm{~K}$ in a $\left(\lambda^{-1} \kappa_{0}, \lambda^{-1} \tau_{0}\right)$-plane calculated from eq 4 is shown in Figure 2, the attached numbers indicating the values of $10^{3} \mathrm{~d} \ln \left\langle R^{2}\right\rangle_{0} / \mathrm{d} T$ (in $\mathrm{deg}^{-1}$ ). Along the heavy solid curve (with the number 0 attached), it vanishes at all temperatures. The numbers in parentheses indicate the observed values given in Table III for a-PS and a-P $\alpha$ MS. It is interesting to see from a comparison of Figure 2 with Figure 4.13 of ref 1 or Figure 2 of ref 31 that the temperature coefficient becomes positive for typical HW chains having strong helical nature. However, this requires a remark. In general, such a chain with a strong helical nature is locally curved at low temperatures but, to some extent, stretches itself out when thermal agitation increases with increasing temperature. At higher temperatures, the temperature coefficient tends, of course, to negative values, since both $\lambda^{-1} \kappa_{0}$ and $\lambda^{-1} \tau_{0}$ vanish in the limit of $T \rightarrow \infty\left(\lambda^{-1} \rightarrow 0\right)$.

\section{CONCLUSIONS}

The temperature coefficient $\mathrm{d} \ln \left\langle R^{2}\right\rangle_{0} / \mathrm{d} T$ was determined from light scattering measurements for a-PS with $f_{\mathrm{r}}=0.59$ and a-P $\alpha \mathrm{MS}$ with $f_{\mathrm{r}}=0.72$, both in toluene and in the range of temperature 15.0$55.0^{\circ} \mathrm{C}$, on the assumption that the excluded-volume effect in good solvents is independent of $T$. It has then been shown that the coefficient is definitely negative for a-PS (with a weak helical nature) but almost vanishes for a-P $\alpha \mathrm{MS}$ (with a strong helical nature). The results are consistent with the HW theory prediction.

Acknowledgment. This research was supported in part by the 21st century COE program "COE for a United Approach to New Materials Science" from the Ministry of Education, Culture, Sports, Science, and Technology, Japan.

\section{REFERENCES}

1. H. Yamakawa, "Helical Wormlike Chains in Polymer Solutions," Springer, Berlin, 1997. 
2. H. Yamakawa, Polym. J., 31, 109 (1999).

3. T. Konishi, T. Yoshizaki, J. Shimada, and H. Yamakawa, Macromolecules, 22, 1921 (1989), and succeeding papers.

4. Y. Tamai, T. Konishi, Y. Einaga, M. Fujii, and H. Yamakawa, Macromolecules, 23, 4067 (1990), and succeeding papers.

5. F. Abe, Y. Einaga, and H. Yamakawa, Macromolecules, 24, 4423 (1991).

6. T. Yamada, T. Yoshizaki, and H. Yamakawa, Macromolecules, 25, 1487 (1992), and succeeding papers.

7. M. Kamijo, N. Sawatari, T. Konishi, T. Yoshizaki, and H. Yamakawa, Macromolecules, 27, 4259 (1994), and succeeding papers.

8. M. Osa, T. Yoshizaki, and H. Yamakawa, Macromolecules, 33, 4828 (2000), and succeeding papers.

9. H. Yamakawa and T. Yoshizaki, Macromolecules, 15, 1444 (1982).

10. P. J. Flory, "Statistical Mechanics of Chain Molecule," Interscience, New York, 1969.

11. J. W. Mays, N. Hadjichristidis, and L. J. Fetters, Macromolecules, 18, 2231 (1985).

12. J. M. Mays, N. Hadjichristidis, W. W. Graessley, and L. J. Fetters, J. Polym. Sci., Part B, 24, 2553 (1986).

13. W. Burchard, Makromol. Chem., 50, 20 (1960).

14. W. H. Stockmayer and M. Fixman, J. Polym. Sci., Part C, 1, 137 (1963).

15. M. Osa, M. Sumida, T. Yoshizaki, H. Yamakawa, K. Ute, T. Kitayama, and K. Hatada, Polym. J., 32, 361 (2000).

16. F. Abe, Y. Einaga, T. Yoshizaki, and H. Yamakawa, Macromolecules, 26, 1884 (1993).

17. F. Abe, Y. Einaga, and H. Yamakawa, Macromolecules, 26,
1891 (1993).

18. H. Yamakawa, F. Abe, and Y. Einaga, Macromolecules, 26, 1898 (1993).

19. T. Arai, F. Abe, T. Yoshizaki, Y. Einaga, and H. Yamakawa, Macromolecules, 28, 3609 (1995).

20. T. Arai, F. Abe, T. Yoshizaki, Y. Einaga, and H. Yamakawa, Macromolecules, 28, 5458 (1995).

21. Gj. Deželić and J. Vavra, Croat. Chem. Acta, 38, 35 (1966).

22. D. N. Rubingh and H. Yu, Macromolecules, 9, 681 (1976).

23. G. C. Berry, J. Chem. Phys., 44, 4550 (1966).

24. M. Osa, Y. Ueno, T. Yoshizaki, and H. Yamakawa, Macromolecules, 34, 6402 (2001).

25. F. D. Rossini, K. S. Pitzer, R. L. Arnett, R. M. Braun, and G. C. Pimentel, "Selected Values of Physical and Thermodynamic Properties of Hydrocarbons and Related Compounds," Carnegie Press, Pittsburgh, 1953.

26. B. L. Johnson and J. Smith, "Light Scattering from Polymer Solutions," M. B. Huglin, Ed., Academic Press, London, 1972, Chap. 2.

27. H. Yamakawa, "Modern Theory of Polymer Solutions," Harper \& Row, New York, 1971. Its electronic edition is available on-line at the URL: http://www.molsci.polym. kyoto-u.ac.jp/archives/redbook.pdf

28. D. A. McQuarrie, "Statistical Mechanics," Harper \& Row, New York, 1973, Chap. 12.

29. O. Kratky and G. Porod, Recl. Trav. Chim. Pays-Bas, 68, 1106 (1949).

30. T. Konishi, T. Yoshizaki, T. Saito, and H. Yamakawa, Macromolecules, 23, 290 (1989).

31. H. Yamakawa, J. Shimada, and M. Fujii, J. Chem. Phys., 68 , 2140 (1978). 